\title{
P I 9- 18. Programme EVA centre for AIDS reagents: a resource for research and reference reagents and materials sharing
}

\section{Ramaswamy}

\author{
Address: Programme EVA Centre for AIDS Reagents, NIBSC, Potters Bar, UK \\ from AIDS Vaccine 2009 \\ Paris, France. 19-22 October 2009 \\ Published: 22 October 2009 \\ Retrovirology 2009, 6(Suppl 3):P338 doi:10.1 I86/I742-4690-6-S3-P338
}

This abstract is available from: http://www.retrovirology.com/content/6/S3/P338

(C) 2009 Ramaswamy; licensee BioMed Central Ltd.

\section{Background}

Programme EVA Centre for AIDS Reagents (EVA CFAR) is a reagent resource that provides well characterised research materials and standards to the HIV/AIDS community. The reagent programme is one of the longest continually funded programmes in Europe and supports projects based on the discovery and development of HIV/ AIDS vaccines and other prevention strategies. Although considerable resources have been contributed by industry, government and academia to this end, identification of an efficacious HIV/AIDS vaccine has yet to be accomplished. In this effort, EVA CFAR continues to support basic, preclinical and clinical research for therapeutic and preventative strategies against HIV/AIDS.

\section{Methods}

Over the last two decades $>4200$ reagents have been deposited, several acquired through project partnerships. EVA CFAR actively participates in EC-supported projects including (i) FP6 Specific Support Action (Neutnet); (ii) FP6 AIDS Vaccine Integrated Project; (iii) FP6 European Vaccines and Microbicides Enterprise (Network of Excellence); (iv) FP7 project Next Generation HIV-1 Immunogens that induce broadly reactive neutralizing antibodies. The repository also collaborates with the Bill and Melinda Gates-funded project that is part of the Collaboration for AIDS Vaccine Discovery consortium.

\section{Results}

The repository has long provided researchers with specialised reagents, many which are commercially unavailable and require customised production. The inventory available at http://www.nibsc.ac.uk/spotlight/aids.html includes cell lines, virus isolates, molecular clones, monoclonal/polyclonal antibodies, peptides, recombinant proteins and international standards and working rea- gents for antibody and nucleic acid-based assays. The enlargement of the repository is very much reliant on reagents being donated from individual laboratories, however if a resource is needed in a specific research area please contact Dr Meghna Ramaswamy Meghna.Ramaswamy@nibsc.hpa.org.uk or Mrs Sarah Goriup Sarah.Goriup@nibsc.hpa.org.uk for information.

\section{Conclusion}

Programme EVA CFAR supports and facilitates the exchange of reagents to new and promising areas of work and encourages collaborative research and efforts aimed at standardisation of reagents and methodologies. 